\title{
HLA-G as a target molecule in specific immunotherapy against renal cell carcinoma
}

\author{
YOSHIHIRO KOMOHARA ${ }^{1,2,6}$, MAMORU HARADA $^{2,3}$, YUKI ISHIHARA $^{2}$, SHIGETAKA SUEKANE $^{4}$, \\ MASANORI NOGUCHI ${ }^{4}$, AKIRA YAMADA ${ }^{1,5}$, KEI MATSUOKA ${ }^{4}$ and $\mathrm{KYOGO} \mathrm{ITOH}^{2,5}$ \\ ${ }^{1}$ Cancer Vaccine Development Division, Kurume University Research Center for Innovative Cancer \\ Therapy, Kurume University School of Medicine; ${ }^{2}$ Department of Immunology, Kurume University \\ School of Medicine, Kurume; ${ }^{3}$ Department of Immunology, Shimane University Faculty of Medicine, \\ Shimane; ${ }^{4}$ Department of Urology, Kurume University School of Medicine; ${ }^{5}$ Center of the \\ 21 st Century COE Program for Medical Science, Kurume University, Kurume, Japan
}

Received July 10, 2007; Accepted August 24, 2007

\begin{abstract}
The human leukocyte antigen (HLA)-G molecule can exert immunoregulatory functions. However, its limited tissue distribution and preferential expression in a variety of malignancies suggest the possibility that it could be a target in anti-cancer immunotherapy. In the present study, we tested this possibility by focusing on renal cell carcinoma (RCC) patients, especially those with HLA-A24 alleles. Four HLA-Gderived peptides were prepared based on the binding motif to the HLA-A24 alleles. After a stabilization assay confirmed the binding of these peptides to HLA-A24 molecules, they were screened for the capacity to induce peptide-specific cytotoxic T lymphocytes (CTLs) from peripheral blood mononuclear cells (PBMCs) of HLA-A24+ RCC cancer patients. As a result, the HLA- $G_{146-154}$ peptide was found to effectively induce peptide-specific CTLs, and HLA-G 146-154 $_{\text {peptide-stimulated }}$ PBMCs exhibited cytotoxic activity against HLA-G-expressing HLA-A24+ RCC cells. Antibody blocking and cold inhibition experiments confirmed that the cytotoxicity was partially ascribed to peptide-specific and HLA class I-restricted CD8 ${ }^{+}$ $\mathrm{T}$ cells. These results indicate that HLA-G-associated immunoregulation can be overcome and that HLA-G peptide-based anti-cancer immunotherapy is feasible.
\end{abstract}

\section{Introduction}

Renal cell carcinoma ( $\mathrm{RCC}$ ) is resistant to conventional chemotherapy, and immunotherapy is considered a promising

Correspondence to: Dr Yoshihiro Komohara, Present address: ${ }^{6}$ Department of Cell Pathology, Graduate School of Medical Sciences, Kumamoto University, 1-1-1 Honjo Kumamoto, Kumamoto 860-8556, Japan

E-mail: ycomo@kumamoto-u.ac.jp

Key words: HLA-G, renal cell carcinoma, immunotherapy, HLAA24, peptide treatment modality because some patients with metastatic RCC have shown favorable responses to immunotherapy (1-3). Previously, a panel of cancer-associated antigens has been identified, enabling us to perform specific immunotherapy (4). We have also identified a number of cancer-associated antigens recognized by epithelial cancer-reactive cytotoxic $\mathrm{T}$ lymphocytes (CTLs) (5-7), and some of these antigens are applicable to RCC patients (8-10). However, cancer cells escape from the immune system through several mechanisms: loss of tumor antigen, down-regulation of human leukocyte antigen (HLA) class I antigen, defective death receptor signaling, and regulatory T cells. In addition, several immunosuppressive molecules and factors, including interleukin (IL)-10, transforming growth factor- $\beta$, prostaglandin E2, arginase-I, and indoleamine 2,3-dioxygenase, are involved in immunosuppression in the tumor-bearing state (11).

HLA-G, a nonclassical HLA class I molecule, is known to inhibit anti-cancer immune responses. HLA-G inhibits CTL responses and protects HLA class I-deficient targets from NK-mediated lysis through interactions with killer inhibitory receptors on NK cells (12-14). On the other hand, HLA-G exhibits a limited tissue distribution (15). In addition, this molecule is preferentially expressed in a variety of malignancies, including lung cancer, breast cancer, mesothelioma, cervical cancer, choriocarcinoma, bladder cancer, and RCC (16-21). In RCC, 10 of 37 RCC samples expressed high levels of HLA-G protein, whereas normal kidney cells lack HLA-G expression (21). These lines of evidence suggest the possibility that HLA-G could be a target molecule in anticancer immunotherapy. Elimination of HLA-G-expressing refractory cancer cells is inevitable for successful anti-cancer therapy. With this in mind, we tested the possibility of immuno-therapy targeting HLA-G by focusing on HLA$\mathrm{A} 24^{+} \mathrm{RCC}$ patients.

\section{Materials and methods}

Patients. The study protocol (Protocol \# 2484) was approved by the Institutional Ethics Review Board of Kurume 
Table I. HLA-G-derived peptide candidates binding to the HLA-A*2402 molecules.

\begin{tabular}{|c|c|c|c|c|c|}
\hline \multirow[b]{2}{*}{ Peptide } & \multirow[b]{2}{*}{ Sequence } & \multicolumn{2}{|c|}{ Binding score } & \multicolumn{2}{|c|}{ Stabilization assay (MFI) } \\
\hline & & BIMAS & SYFPEITHI & $10 \mathrm{mM}$ & $100 \mathrm{mM}$ \\
\hline HLA-G 146-154 & DYLALNEDL & 360 & 23 & 43 & 48 \\
\hline HLA-G 194-202 & RYLENGKEM & 99 & 14 & 81 & 102 \\
\hline HLA-G 139-148 & RYAYDGKDYL & 200 & 20 & 47 & 65 \\
\hline HLA-G 141-150 & AYDGKDYLAL & 720 & 25 & 42 & 51 \\
\hline EBV-A24 & TYGPVFMCL & 403 & 24 & 80 & 102 \\
\hline EBV-A2 & GLCTLVAML & 5 & 11 & 5 & 7 \\
\hline
\end{tabular}

The entire amino acid sequence was scanned using epitope prediction algorithm in the following web sites: BIMAS, http://wye.cit.nih.gov/ molbio/hla_bind/; SYFPEITHI, http://www.syfpeithi.de/. Stabilization assay was performed as described in the Materials and methods. MFI, mean fluorescence intensity.

University. Informed written consent was obtained from all the subjects from whom peripheral blood mononuclear cells (PBMCs) were taken. PBMCs were prepared by FicollConray density gradient centrifugation and cryo-preserved until they were used for the experiments. The expression of HLA-A24 molecule on PBMCs of cancer patients was determined by flow cytometry as described previously (22).

Cell lines. All cell lines used were incubated in RPMI-1640, supplemented with $10 \%$ fetal bovine serum (FBS). MAMIYA is an HLA-A24+ and HLA-G- RCC, and an HLAG-expressing MAMIYA, designated as MAMIYA-HLAG, was established by the stable transfection with HLA-G genecontaining pcDNA3.1 using FuGene (Roche, Mannheim, Germany), followed by the culture with geneticin $(1 \mathrm{mg} / \mathrm{ml})$. RMA-S transfectant cells expressing the $H L A-A * 2402$ gene, designated as RMA-S-A*2402, were generated by the transfection with the $H L A-A * 2402$ gene into RMA-S cells, followed by the culture with geneticin $(1 \mathrm{mg} / \mathrm{ml})$. C1R-A24 is an HLA-A*2402-expressing C1R subline (Dr M. Takiguchi, Kumamoto University, Japan).

Immuno-staining and flow cytometry. HLA-G protein expression on MAMIYA-HLAG cells were detected by mouse anti-HLA-G antibody (MEM-G/9, Abcam). The cells were fixed in cold acetone, then incubated with MEM-G/9 or control mouse IgG. After washing in PBS, the cells were incubated with HRP-labeled goat anti-mouse antibody (Nichirei; Tokyo, Japan). The reaction was visualized by use of the DAB substrate system (Dako). Paraffin-embedded RCC samples were deparaffinized in xylene and rehydrated in ethanol, and then antigens were retrieved by microwave for $10 \mathrm{~min}$ in $10 \mathrm{mM}$ sodium citrate ( $\mathrm{pH} \mathrm{6.0)}$ ). The sections were reacted with mouse anti-HLA-G antibody (MEM-G/1, Abcam), and then incubated with HRP-labeled goat antimouse antibody (Nichirei). The reaction was visualized by use of the DAB substrate system (Dako). Slides were counterstained with hematoxylin. For flow cytometry, the cells were reacted with MEM-G/9 or control mouse IgG. The Alexa Fluor 488 goat anti-mouse IgG (Invitrogen,
Carlsbad, CA) was used as the secondary antibody. The cells were analyzed using EPICS (Beckman Coulter, Miami, FL).

Peptides and stabilization assay. All peptides used were purchased from Genenet (Fukuoka, Japan), and their amino acid sequences are shown in Table I. Peptide binding to HLA-A*2402 molecules was examined using the stabilization assay according to a previously reported method with several modifications (23). Briefly, RMA-S-A*2402 cells $\left(5 \times 10^{5}\right.$ cells per well in a 24-well plate) were cultured at $26^{\circ} \mathrm{C}$ for $20 \mathrm{~h}$ in RPMI-1640 supplemented with $20 \%$ FBS, followed by incubation with Opti-MEM (Invitrogen) containing 10 or $100 \mu \mathrm{M}$ peptide and human $\beta_{2}$ microglobulin $(2 \mathrm{ng} / \mathrm{ml})$ at $26^{\circ} \mathrm{C}$ for $2 \mathrm{~h}$, and then cultured at $37^{\circ} \mathrm{C}$ for $3 \mathrm{~h}$. After washing with PBS, these cells were put on ice for $30 \mathrm{~min}$ with antiMHC class I mAb, PT85A (VMRD, Pullman, WA). After washing twice with PBS, these cells were put on ice for $30 \mathrm{~min}$ with Alexa Fluor 488 goat anti-rabbit IgG (Invitrogen). An EBV-derived peptide binding to HLA-A24 molecules, designated as EBV-A24, was used as a positive control, and an EBV-derived peptide binding to HLA-A2 molecules, designated as EBV-A2, was used as a negative control.

Induction of peptide-specific CTLs from PBMCs. Influenza (Flu) virus, EB virus, and HIV-derived peptides were used as controls binding to HLA-A24 alleles, as described previously (8-10). In vitro induction of peptide-specific CTLs was performed according to a previously reported method with several modifications (24). Briefly, PBMCs were incubated with $10 \mu \mathrm{g} / \mathrm{ml}$ of each peptide in quadruplicate in a 96-well microplate (Nunc, Roskilde, Denmark). The culture medium consisted of 45\% RPMI-1640, 45\% AIM-V medium (Life Technologies, Gaithersburg, MD), 10\% FBS, 100 units/ml IL-2, and 0.1 mM MEM non-essential amino acid solution (Life Technologies). On the 15th day of culture, cells were separated into four wells, and then cultured with the corresponding peptide-pulsed C1R-A24 cells. The HIV peptide was used a negative control. After an 18-h incubation, the IFN- $\gamma$ production was determined by ELISA. 
Table II. Induction of peptide-specific CTLs from the PBMCs of HLA-A24+ RCC patients.

\begin{tabular}{|c|c|c|c|c|c|c|c|}
\hline \multirow[b]{3}{*}{ Patient } & \multicolumn{6}{|c|}{$\mathrm{IFN}-\gamma(\mathrm{pg} / \mathrm{ml})$} & \multirow{3}{*}{$\begin{array}{l}\text { HLA-G expression } \\
\text { in cancer tissue }\end{array}$} \\
\hline & \multicolumn{4}{|c|}{ HLA-G peptide } & \multirow[b]{2}{*}{ Flu } & \multirow[b]{2}{*}{ EBV } & \\
\hline & $146-154$ & 194-202 & $139-148$ & $141-150$ & & & \\
\hline Pt. 1 & $\underline{83}$ & 8 & $\underline{64}$ & 39 & $\underline{109}$ & $\underline{186}$ & + \\
\hline Pt. 2 & 17 & 7 & 16 & 17 & $\underline{108}$ & $\underline{125}$ & + \\
\hline Pt. 3 & $\underline{143}$ & 3 & 0 & 0 & 59 & 22 & + \\
\hline Pt. 4 & 0 & 46 & 26 & $\underline{162}$ & $\underline{89}$ & 20 & + \\
\hline Pt. 5 & $\underline{93}$ & 47 & 20 & 14 & $\underline{199}$ & $\underline{298}$ & - \\
\hline Pt. 6 & 29 & 15 & 0 & 9 & $\underline{142}$ & 19 & - \\
\hline Pt. 7 & $\underline{115}$ & 30 & $\underline{177}$ & 55 & $\underline{79}$ & $\underline{109}$ & - \\
\hline Pt. 8 & 0 & 37 & 9 & 15 & N.D. & N.D. & - \\
\hline Pt. 9 & 0 & 0 & $\underline{65}$ & 1 & 67 & 23 & N.E. \\
\hline Pt. 10 & $\underline{51}$ & 7 & $\underline{70}$ & $\underline{63}$ & $\underline{108}$ & 50 & - \\
\hline Total & $(5 / 10)$ & $(0 / 10)$ & $(3 / 10)$ & $(2 / 10)$ & $(7 / 9)$ & $(4 / 9)$ & $(4 / 9)$ \\
\hline
\end{tabular}

The PBMCs were stimulated in vitro with each of the indicated peptides, and peptide-specific reactivity was examined. The results were evaluated by the two-tailed Student's t-test $(\mathrm{P}<0.05)$, and the positive results are underlined. N.D., not done. HLA-G expression in RCC tissues were examined by immunohistochemistry. Samples were judged to be positive if $>10 \%$ of the tumor cells showed moderate or strong immunoreaction. NE, not examined.

Cytotoxicity assay. After the repeated stimulation with a corresponding peptide for 15 days, these cultured cells were cultured in the presence of 100 units/ml IL-2 alone for further 10-14 days. Then, the cultured cells were tested for their cytotoxicity against MAMIYA and MAMIYA-HLAG cells by a standard 6-h ${ }^{51} \mathrm{Cr}$-release assay. Phytohemagglutinin (PHA)-activated T cells from HLA-A24+ healthy donors were used as a control. Peptide-stimulated PBMCs or purified $\mathrm{CD} 8^{+} \mathrm{T}$ cells were used as effector cells. $\mathrm{CD} 8^{+} \mathrm{T}$ cells were positively isolated using a CD8-positive isolation kit (Dynal, Oslo, Norway). Two thousand ${ }^{51} \mathrm{Cr}$-labeled cells per well were cultured with effector cells in 96-round-well plates, and then the specific ${ }^{51} \mathrm{Cr}$-release was calculated as described previously (22). When peptide-stimulated PBMCs were used as effector cells, K562 cells were added into wells to prevent non-specific cytotoxicity of NK cells. In an experiment, either anti-HLA class I mAb (W6/32) or anti-CD14 mAb, as an isotype-matched control, was added into wells before the ${ }^{51} \mathrm{Cr}$-release assay to confirm HLA class I restriction. The specificity of peptide-stimulated PBMCs was confirmed by cold inhibition assay. Twenty thousand unlabeled C1R-A24 cells, which were pre-pulsed with either a corresponding HLA-G peptide or the HIV peptide, were used as cold target cells (22).

\section{Results}

Four HLA-G peptides binding to the HLA-A*2402 molecules. First, we prepared four HLA-G peptides based on the binding motifs using the BIMAS and SYFPEITHI web sites (Table I). We then conducted the HLA stabilization assay using RMAS-A*2402 cells to directly confirm that these four peptides can bind to the HLA-A*2402 molecules. RMA-S is a mouse mutant cell line that is deficient in antigen processing (25), and its HLA class I transfectant is useful for the stabilization assay (23). RMA-S-A*2402 is a subline that was stably transfected with the $H L A-A * 2402$ gene. EBV-A24 and EBVA2 peptides were used as positive and negative controls, respectively. As shown in Table I, the expression levels of HLA-A24 molecules when pulsed with HLA-G-derived peptides at a dose of either 10 or $100 \mu \mathrm{M}$ were higher than those when pulsed with the negative control EBV-A2 peptide. Their levels were comparable to those of the positive control EBV-A24 peptide. These results indicate that four HLA-G peptides can bind to the HLA-A*2402 molecules and have the potential to induce peptide-specific CTLs.

Induction of HLA-G peptide-specific CTLs from the PBMCs of HLA-A24+ RCC patients and healthy donors. We next determined whether or not the four HLA-G peptides could induce peptide-specific CTLs from the PBMCs of HLA-A24+ RCC patients or healthy donors. The PBMCs were stimulated in vitro with each HLA-G peptide or control peptide, and were examined for their IFN- $\gamma$ production in response to corresponding peptide-pulsed C1R-A24 cells. As a result, the HLA-G ${ }_{146-154}$, HLA-G ${ }_{194-202}$, HLA-G $_{139-148}$, and HLA-G 141-150 $_{1}$ peptides induced corresponding peptide-reactive CTLs from the PBMCs of 5, 0, 3, and 2 of 10 HLA-A24+ RCC patients, respectively (Table II). The Flu and EBV peptides induced peptide-specific CTLs from the PBMCs of 7 and 4 of 9 HLA-A $24^{+}$RCC patients, respectively. We also examined the HLA-G expression in RCC tissues of 9 patients to see whether or not there is a correlation between the induction of HLA-G peptide-specific CTLs and HLA-G expression in 
Table III. Induction of peptide-specific CTLs from the PBMCs of HLA-A24+ healthy donors.

\begin{tabular}{|c|c|c|c|c|c|c|}
\hline \multirow{3}{*}{$\begin{array}{l}\text { Healthy } \\
\text { donors }\end{array}$} & \multicolumn{6}{|c|}{$\mathrm{IFN}-\gamma(\mathrm{pg} / \mathrm{ml})$} \\
\hline & \multicolumn{4}{|c|}{ HLA-G peptide } & \multirow[b]{2}{*}{ Flu } & \multirow[b]{2}{*}{ EBV } \\
\hline & $146-154$ & 194-202 & $139-148$ & $141-150$ & & \\
\hline HD 1 & 0 & 39 & $\underline{92}$ & 26 & $\underline{740}$ & 20 \\
\hline HD 2 & $\underline{78}$ & 0 & 0 & 19 & 20 & 22 \\
\hline HD 3 & 0 & 13 & 6 & 0 & $\underline{82}$ & 17 \\
\hline HD 4 & 6 & 0 & 0 & 0 & 47 & 9 \\
\hline HD 5 & 0 & 0 & $\underline{106}$ & $\underline{62}$ & 40 & $\underline{56}$ \\
\hline Total & $(1 / 5)$ & $(0 / 5)$ & $(2 / 5)$ & $(1 / 5)$ & $(2 / 5)$ & $(1 / 5)$ \\
\hline
\end{tabular}

The PBMCs were stimulated in vitro with each of the indicated peptides, and peptide-specific reactivity was examined. The results were evaluated by the two-tailed Student's t-test $(\mathrm{P}<0.05)$, and the positive results are underlined.

A

MAMIYA

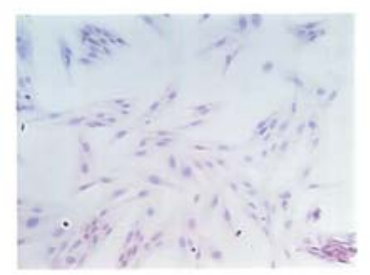

$\mathbf{B}$

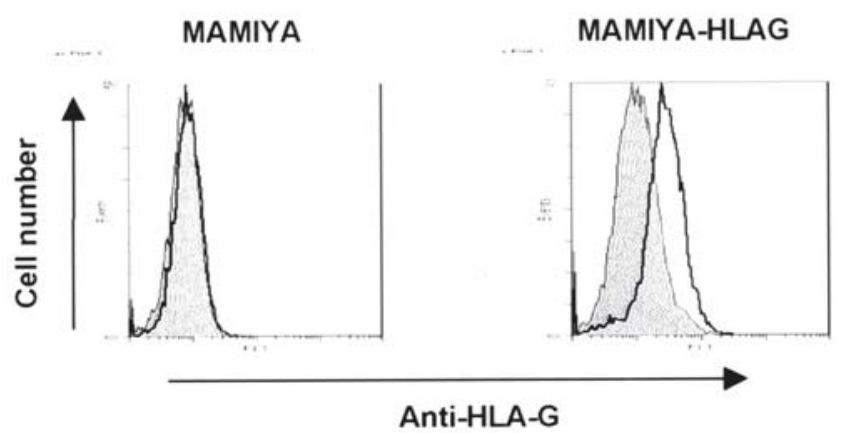

Figure 1. HLA-G-expressing RCC cell line. (A) Immunocytostaining was performed on MAMIYA and MAMIYA-HLAG. (B) These two cell lines were stained with anti-HLA-G mAb followed by Alexa Fluor 488-conjugated anti-mouse IgG, and were then analyzed by flow cytometry.

cancer tissues. We detected HLA-G expression in cancer tissues of 4 patients, but the induction of HLA-G peptidespecific CTLs was possible even from the PBMCs of RCC patients whose cancer tissues were negative for HLA-G protein. We also determined whether or not HLA-G peptidespecific CTLs could be induced from the PBMCs of HLAA $24^{+}$healthy donors, and found that these four HLA-G peptides induced corresponding peptide-reactive CTLs from the PBMCs of $1,0,2,1$, and 0 of 5 HLA-A $24^{+}$healthy donors, respectively (Table III). In contrast to the cases with RCC patients, the HLA-G $\mathrm{G}_{146-154}$ peptide was not the best candidate to induce peptide-specific CTLs from the PBMCs of HLA-A $24^{+}$healthy donors. These results indicate that the HLA-G ${ }_{146-154}$ peptide is useful for inducing peptide-specific CTLs in HLA-A24+ RCC patients.

Cytotoxicity of HLA-G $G_{146-154}$ peptide-stimulated PBMCs against $H L A-G$-expressing RCC cells. To examine whether or not HLA-G peptide-stimulated PBMCs could exhibit cytotoxicity against RCC cells expressing HLA-G protein, the HLA-G1 gene was stably transfected into HLA-A24+ MAMIYA cells, designated MAMIYA-HLAG. The expression of HLA-G protein was confirmed by both immunocytostaining and flow cytometry (Fig. 1A and B).

We next determined whether or not HLA- $\mathrm{G}_{146-154}$ peptidestimulated PBMCs induced from the PBMCs of HLA-A24+ RCC patients could show cytotoxicity against MAMIYAHLAG cells (Fig. 2A). HLA-G ${ }_{146-154}$ peptide-stimulated PBMCs of patients 5 and 7 showed higher levels of cytotoxicity against MAMIYA-HLAG cells than against MAMIYA cells or PHA-stimulated T-cell blasts. These results indicate that $\mathrm{HLA}-\mathrm{G}_{146-154}$ peptide-specific CTLs exhibited cytotoxicity against HLA-G-expressing HLA-A24+ RCC cells.

CD8 ${ }^{+}$T-cell-dependent and peptide-specific cytotoxicity against MAMIYA-HLAG cells. We further attempted to identify cells responsible for the cytotoxicity of HLA-G $\mathrm{G}_{146-154}$ peptide-stimulated PBMCs against HLA-G-expressing RCC cells. HLA-G $\mathrm{G}_{146-154}$ peptide-stimulated cells were positively isolated for $\mathrm{CD}^{+} \mathrm{T}$ cells before the cytotoxicity assay. Antipan HLA class I mAb (W6/32) or control anti-CD14 mAb was added before the cytotoxic assay. Fig. $2 \mathrm{~B}$ shows that the cytotoxicity of HLA-G $\mathrm{G}_{146-154}$ peptide-stimulated $\mathrm{CD}^{+} \mathrm{T}$ cells against MAMIYA-HLAG cells was significantly inhibited by the addition of anti-HLA class I mAb, but not by the addition of control anti-CD14 mAb. In addition, cytotoxicity was significantly suppressed by the addition of HLA-G $\mathrm{G}_{146-154}$ peptide-pulsed unlabeled C1R-A24 cells, but not by the addition of control HIV peptide-pulsed unlabeled C1R-A24 
A

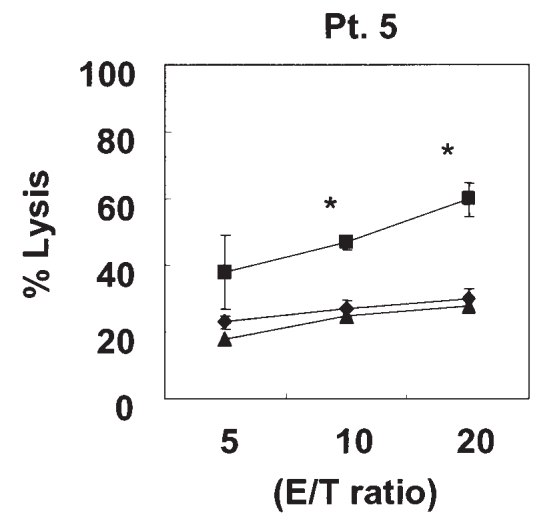

B

Antibody added

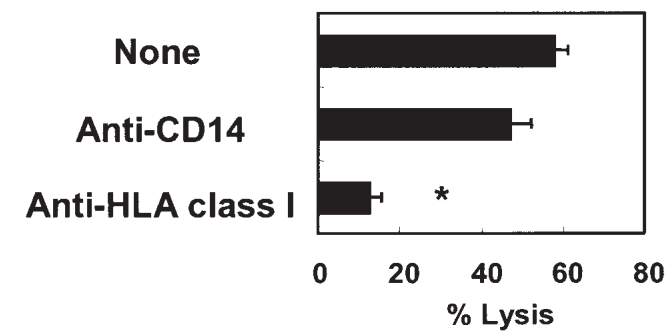

Pt. 7

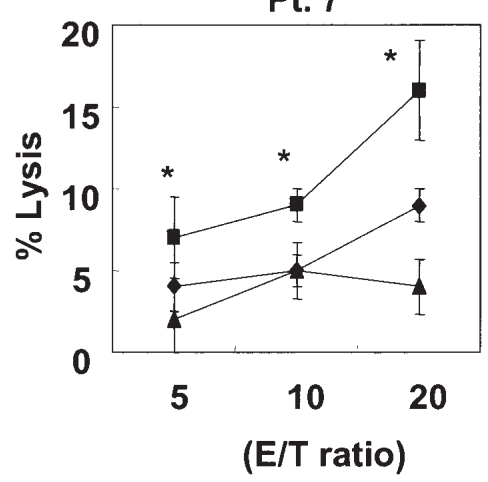

C

Figure 2. Cytotoxicity of peptide-stimulated PBMCs against HLA-G-expressing RCC cells. (A) PBMCs of 2 HLA-A24+ RCC patients were in vitro stimulated with the HLA-G peptide and examined for their cytotoxicity against MAMIYA, MAMIYA-HLAG, and PHA-activated T-cell blasts. (B) Indicated anti-HLA class I and control (anti-CD14) monoclonal antibodies were added into wells before the cytotoxic assay. The cytotoxicity of peptidestimulated $\mathrm{CD}^{+} \mathrm{T}$ cells from patient 5 was significantly inhibited by the addition of anti-HLA class I mAb, but not by that of control mAb. (C) Cytotoxicity of peptide-stimulated $\mathrm{CD}^{+} \mathrm{T}$ cells from patient 5 against MAMIYA-HLAG cells was examined in the presence of HLA-G $\mathrm{H}_{146-154}$ peptide-pulsed unlabeled C1R-A24 cells (cold target) or control HIV peptide-pulsed unlabeled C1R-A24 cells. ${ }^{*} \mathrm{P}<0.05$ statistically significant (Student's t-test).

cells (Fig. 2C). These results indicate that the cytotoxicity against HLA-A24+ and HLA-G-expressing RCC cells was partially dependent on HLA class I-restricted and peptidespecific $\mathrm{CD}^{+} \mathrm{T}$ cells.

\section{Discussion}

Although molecular targeting therapies have been developed, immunotherapy is a promising alternative modality for the treatment of RCC (3). Nevertheless, effective specific immunotherapy against RCC has not been established yet, and one plausible reason is the limited information on target antigens of RCC. In this study, we tested the possibility that HLA-G could be a target molecule in specific immunotherapy against $\mathrm{RCC}$ in spite of its immunoregulatory functions.

Certain cancer cells resist anti-tumor immune responses by expressing immunoregulatory molecules or anti-apoptotic genes. However, from a therapeutic standpoint, elimination of these therapy-resistant cells is inevitable for successful anti-cancer therapy. Previous studies have revealed that antiapoptotic or immunoregulatory molecules can be targets in specific immunotherapy. A cancer vaccine targeting survivin, an inhibitor of apoptosis protein, has been developed (26), and an HLA-A2-restricted $\mathrm{Bcl} 2$-derived epitope on tumors recognized by CTLs has been identified (27). We have also reported that serine protein inhibitor 9 can be recognized by CTLs of epithelial cancer patients (28), and that immediate early response gene X-1, a stress-inducible anti-apoptotic gene, encodes CTL epitopes capable of inducing HLA-A33restricted and tumor-reactive CTLs in gastric cancer patients (30). These lines of evidence indicate that immunoregulatory molecule-derived epitope peptides could be recognized by CTLs and might be a target in specific immunotherapy when their tissue distribution is limited and preferentially expressed in malignancies. We therefore undertook this study. As a result, we identified that the HLA-G $\mathrm{G}_{146-154}$ peptide can efficiently induce peptide-specific and RCC-reactive CTLs from PBMCs of HLA-24+ RCC patients. We suppose that the HLA-G G $_{146-154}$ peptide is a promising candidate in peptide-based immunotherapy for HLA-A24+ RCC patients.

HLA-G is known to inhibit the cytotoxic activity of not only NK cells but also antigen-specific T lymphocytes. Le Gal et al demonstrated the decreased cytotoxicity of influenzavirus-specific $\mathrm{CD}^{+} \mathrm{T}$ lymphocytes against HLA-G-expressing cells, and that the cytotoxicity was reversed by the addition of anti-HLA-G antibody (13). In the present study, however, HLA-G molecules expressed on MAMIYA-HLAG cells did not inhibit the CTL function and were recognized by peptidespecific CTLs. One explanation for this observation is that the level of surface HLA-G was too low to inhibit T-cell function. 
As shown in Fig. 1A, HLA-G expression in MAMIYA-HLAG cells was prominent in the cytoplasm and less expressed on the cell surface. This idea was also supported by Malmberg et $a l$, who showed that a lower level of HLA-G expression did not impair peptide-specific $\mathrm{CD}^{+} \mathrm{T}$ cells, but that upregulation of HLA-G protein induced by IFN- $\gamma$ resulted in the inhibition of cytotoxic activity of peptide-specific $\mathrm{CD}^{+}$ T cells (26). However, further analysis is needed to elucidate the matter.

In conclusion, we revealed that HLA-G could be a target antigen in specific immunotherapy against HLA-G-expressing RCC. Since HLA-G is preferentially expressed in many malignancies (14-21), our finding might open up the possibility of HLA-G-targeting immunotherapy against a variety of HLA-G-expressing cancers.

\section{Acknowledgements}

This study was supported in part by Grant-in-Aids from the Ministry of Education, Science, Sports and Culture of Japan (KAKENHI: no. 16591628 to K. Matsuoka, no. 16790933 to S. Suekane, no. 18591449 to M. Harada, and no. 12213134 to K. Itoh), and from the Ministry of Health, Labor and Welfare, Japan (no. H14-trans-002, 11-16, and H12-cancer004 to K. Itoh, and no. 15-17 to M. Harada).

\section{References}

1. Selli C, Hinshaw WM, Woodard BH and Paulson DF: Stratification of risk factors in renal cell carcinoma. Cancer 52: 899-903, 1983.

2. Yagoda A, Abi-Rached B and Petrylak D: Chemotherapy for advanced renal-cell carcinoma: 1983-93. Semin Oncol 22: 42-60, 1995.

3. Motzer RJ: Renal cell carcinoma: a priority malignancy for development and study of novel therapies. J Clin Oncol 21: 1193-1194, 2003.

4. Novellino L, Castelli C and Parmiani G: A listing of human tumor antigens recognized by $\mathrm{T}$ cells: March 2004 update. Cancer Immunol Immunother 54: 187-207, 2005.

5. Yamada A, Kawano K, Koga M, Matsumoto T and Itoh K: Multidrug resistance-associated protein 3 is a tumor rejection antigen recognized by HLA-A2402-restricted cytotoxic T lymphocytes. Cancer Res 61: 6459-6466, 2001.

6. Itoh $\mathrm{K}$ and Yamada A: Personalized peptide vaccines: a new therapeutic modality for cancer. Cancer Sci 97: 970-976, 2006.

7. Komohara Y, Harada M, Arima Y, et al: Anti-cancer vaccine candidates in specific immunotherapy for bladder carcinoma. Int J Oncol 29: 1555-1560, 2006.

8. Kawagoe N, Shintaku I, Yutani S, et al: Expression of the SART3 tumor rejection antigen in renal cell carcinoma. J Urol 164: 2090-2095, 2000.

9. Miyagi Y, Imai N, Sasatomi T, et al: Induction of cellular immune responses to tumor cells and peptides in colorectal cancer patients by vaccination with SART3 peptides. Clin Cancer Res 7: 3950-3962, 2001.

10. Komohara Y, Harada M, Arima Y, et al: Identification of target antigens in specific immunotherapy for renal cell carcinoma. J Urol 177: 1157-1162, 2007.
11. Kim R, Emi M, Tanabe K and Arihiko K: Tumor-driven evolution of immunosuppressive networks during malignant progression. Cancer Res 66: 5527-5536, 2006.

12. Pazmany L, Mandelboim O, Vales-Gomez M, et al: Human leukocyte antigen- $\mathrm{G}$ and its recognition by natural killer cells. J Reprod Immunol 43: 127-137,1999.

13. Le Gal FA, Titeau B, Sedilik C, et al: HLA-G-mediated inhibition of antigen-specific cytotoxic T lymphocytes. Int Immunol 11: 1351-1356, 1999.

14. Rouas-Freiss N, Moreau P, Ferrone S and Carosella ED: HLA-G protein in cancer: Do they provide tumor cells with an escape mechanism? Cancer Res 65: 10139-10144. 2005.

15. Onno M, Guillaudeux T, Amiot L, et al: The HLA-G gene is expressed at a low mRNA level in different human cells and tissues. Hum Immunol 41: 79-86, 1994.

16. Ibrahim EC, Guerra N, Lacombe MJT, et al: Tumor-specific up-regulation of the nonclassical class I HLA-G antigen expression in renal carcinoma. Cancer Res 61: 6838-6845, 2001.

17. Urosevic M, Kurrer MO, Kamarashev J, et al: Human leukocyte antigen $\mathrm{G}$ up-regulation in lung cancer associated with highgrade histology, human leukocyte antigen class I loss and interleukin-10 production. Am J Pathol 159: 817-824, 2001.

18. Kleinberg L, Florenes VA, Skrede M, et al: Expression of HLA-G in malignant mesothelioma and clinically aggressive breast carcinoma. Virchows Arch 449: 31-39, 2006.

19. Zhou JH, Ye F, Chen HZ, Zhou CY, Lu WG and Xie X: Altered expression of cellular membrane molecules of HLA-DR, HLA-G and CD99 in cervical intraepithelial neoplasias and invasive squamous cell carcinoma. Life Sci 78: 2643-2649, 2006.

20. El-Chennawi FA, Auf FA, El-Diasty AM, et al: Expression of HLA-G in cancer bladder. Egypt J Immunol 12: 57-64, 2005.

21. BuKur J, Rebmann V, Gross-Wilde H, et al: Functional role of human leukocyte antigen-G up-regulation in renal cell carcinoma. Cancer Res 63: 4107-4111, 2003.

22. Matsueda S, Takedatsu H, Yao A, Tanaka M, Noguchi M, Itoh K and Harada $\mathrm{M}$ : Identification of peptide vaccine candidates for prostate cancer patients with HLA-A3 supertype alleles. Clin Cancer Res 11: 6933-6943, 2005.

23. Takiguchi M, Matsuda T, Tomiyama $\mathrm{H}$ and Miwa K: Analysis of three HLA-A*3303 binding peptide anchors using an HLAA*3303 stabilization assay. Tissue Antigens 55: 296-302, 2000.

24. Hida N, Maeda Y, Katagiri K, Takasu H, Harada M and Itoh K: A simple culture protocol to detect peptide-specific T lymphocyte precursors in the circulation. Cancer Immunol Immunother 51: 219-228, 2002.

25. Esquivel F, Yewdell J and Bennink J: RMA/S cells present endogeneously synthesized cytosolic proteins to class I restricted cytotoxic T lymphocytes. J Exp Med 175: 163-168, 1992.

26. Malmberg KJ, Levitsky V, Norell H, et al: IFN- $\gamma$ protects shortterm ovarian carcinoma cell lines from CTL lysis via CD94/ NKG2A-dependent mechanism. J Clin Invest 110: 1515-1523, 2002.

27. Idenoue S, Hirohashi Y, Torigoe T, et al: A potent immunogenic general cancer vaccine that targets survivin, an inhibitor of apoptosis proteins. Clin Cancer Res 11: 1474-1482, 2005.

28. Wang M, Johansen B, Nissen MH, et al: Identification of an HLA-A*0201 restricted Bcl2-derived epitope expressed on tumors. Cancer Lett 251: 86-95, 2007.

29. Tanaka K, Harashima N, Niiya F, et al: Serine proteinase inhibitor 9 can be recognized by cytotoxici $\mathrm{T}$ lymphocytes of epithelial cancer patients. Jpn J Cancer Res 93: 198-208, 2002.

30. Sasada T, Takedatsu H, Azuma K, et al: Immediate early response gene $\mathrm{X}-1$, a stress-inducible antiapoptotic gene, encodes cytotoxic T-lymphocyte (CTL) epitopes capable of inducing human leukocyte antigen-A33-restricted and tumor-reactive CTLs in gastric cancer patients. Cancer Res 64: 2882-2888, 2004. 